Journal of The Magnetics Society of Japan Vol. 13, Supplement, No. S1 (1989)

(C) 1989 by The Magnetics Society of Japan

\title{
THE EFFECT OF EDDY CURRENT ON THE MAGNETIC RECORDING FIELD \\ IN A SINGLE POLE HEAD
}

\author{
Mitsuo HANO and Masaaki NISHIKAWA
}

Faculty of Enginnering, Yamaguchi University, Tokiwadai, Ube 755, Japan

\begin{abstract}
The effect of the eddy current of a single pole head on the magnetic recording field has been investigated by using a three-dimensional finite-element method. It is indicated that the magnetic recording field upon the pole tip surface is little affected by the eddy current in the head pole.
\end{abstract}

\section{INTRODUCTIN}

The perpendicular magnetic recording system has attracted interest because it can achieve a very high recording density over $200 \mathrm{kBPI}$. In order to realize this recording density, the recording head must have a narrow distribution of the dominant component of the fringing field perpendicular to the medium. A profile of the magnetostatic field of the single pole head was three-dimensionally measured by using a large scale model[1], analytically calculated[2], and numerically calculated by using a two-dimensional finite-element method[3]. In order to realize the above high recording density, the recording frequency must become very high, too. It is expected that the magnetic field of the pole head with high frequency is different from the magnetostatic one. The main pole of the single pole type head is usually made of magnetic alloy having reletively high conductivity. Therefore, as the recording frequency increases, large eddy current will occur in the pole, which might exert influence on the recording field on the pole tip. In this paper, the eddy current distribution in the main pole is analyzed by using a three-dimensional finiteelement program. This program can analyze the eddy current for all frequency range.

\section{D FINITE-ETEMENT METHOD}

The basic equation for the analysis of three-dimensional eddy current is given by

$$
\begin{aligned}
\operatorname{rot} \mu^{-1} \operatorname{rot} \vec{A} & +j \omega \sigma \vec{A} \\
-\omega^{2} \varepsilon \vec{A} & =\vec{J}
\end{aligned}
$$

where $\varepsilon, \mu$ and $\sigma$ are permittivity, permeability and conductivity, respectively. $\vec{J}$ is the electric current source. The magnetic vector potential $\vec{A}$ is defined by

$$
\vec{B}=\operatorname{rot} \vec{A}
$$

The term of the eddy current

$$
\vec{J}_{e}=-j \omega \sigma \vec{A}
$$

appears on the left side of Eq.(1).

To obtain the Galerkin formulation, Eq. (1) is muliplied by a vector test function $\vec{a}$, and integrated over the space. By partial integration, one obtains finally

$$
\begin{aligned}
& \int\left(\operatorname{rot} \vec{a} \cdot \mu^{-1} \operatorname{rot} \vec{A}\right) d \Omega \\
& +j \omega \int \vec{a} \cdot \sigma \vec{A} d \Omega-\omega^{2} \int \vec{a} \cdot \varepsilon \vec{A} d \Omega \\
& =\int \vec{a} \cdot \vec{j} d \Omega
\end{aligned}
$$

The vector potential is approximated by the set of the test functions

$$
\vec{A}=\Sigma \vec{a}_{i} A_{i}
$$

where $A_{i}$ is the unknown parameter of the nodal point $i$. By substituting this approximation into $\mathrm{Eq} .(4)$, one reachs the linear equation as

$$
\left([K]+j \omega[C]-\omega^{2}[M]\right)\{A\}=\{P\}
$$

The matrices of $[\mathrm{K}],[\mathrm{C}]$ and $[\mathrm{M}]$ in $\mathrm{Eq} .(6)$ are large, symmetric and sparse. Eq.(6) is solved by using the complex-conjugate gradient(CG) method [4]. 


\section{HEAD MODEL}

The single pole head model is shown in Fig. 1. The ratio of the length $\mathrm{I}$ to the thickness $T$ of the actual head is usually about $10^{6}$. If the actual head is subdivided into the finite-element meshes, the matrices of Eq.(6) become to ill condition because of the above large ratio. Therefore, Eq. (6) can not be solved so far as the complex-CG method is used. In this paper, the head pole with $5 \mu \mathrm{m}$ thickness, 10 $\mu \mathrm{m}$ width and $20 \mu \mathrm{m}$ length is chosen. Its permeability $\mu$ is assumed to be $1000 \mu_{0}$ and conductivity $\sigma$ to be $6.25 \times 10^{6} \mathrm{~S} / \mathrm{m}$ and $6.25 \times$ $10^{7} \mathrm{~S} / \mathrm{m}$. The exciting current in the coil with the cross section of $1 \mu \mathrm{m} \times 2 \mu \mathrm{m}$ is $4.0 \mathrm{~A}$. The recording frequency $f$ is from $1 \mathrm{MHz}$ to 10OMHz. The field distribution in the neighborhood of the pole tip is investigated. In consideration of the symmetric geometry of the head model, the one quarter finite-element model is shown in Fig.2. The boundary conditions are essential on the $x=0$ plane and $y=0$ plane, natural on the $z=0$ plane, and essential on the other three surfaces. The analytical region did not include a recording medium and an auxiliary pole.

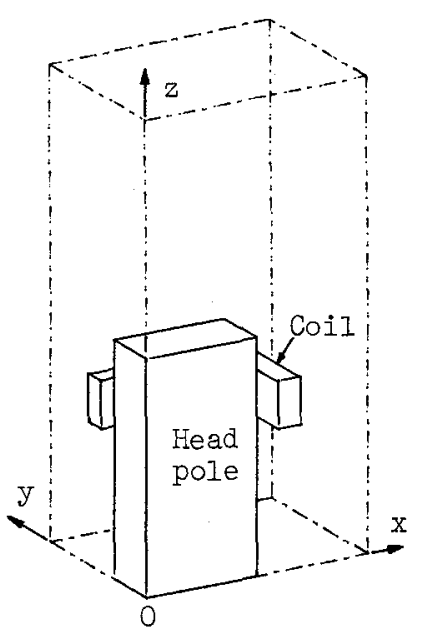

Fig.2 A finite-element modei

\section{RESULTS}

A three-dimensional finite-element analysis of the magnetostatic field is first performed. The distributions of the magnetic field and the magnetic flux density on the $x=0$ plane and $y=0$ plane are shown in Fig.3. It is noted that the magnetic

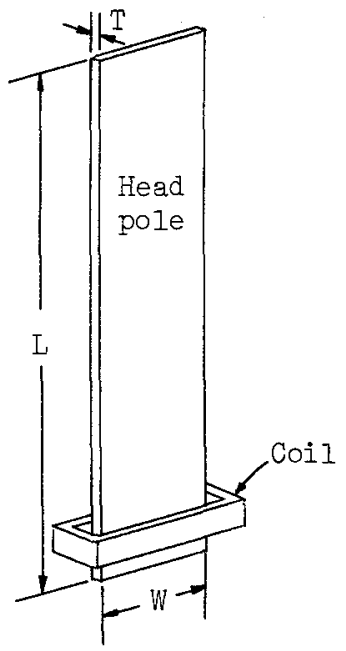

Fig. I A single pole head model

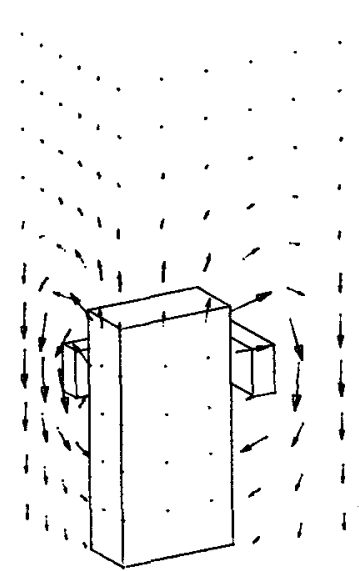

(a) Magnetic field $\mathrm{H}_{\max }=7.76 \times 10^{5} \mathrm{~A} / \mathrm{m}$

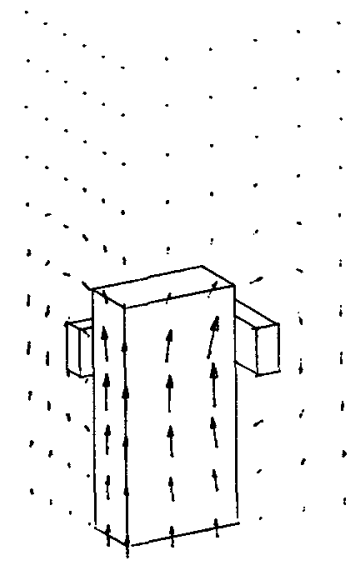

(b) Magnetic flux $\mathrm{B}_{\max }=2.00 \mathrm{~Wb} / \mathrm{m}^{2}$

Fig.3 Distributions of the magnetic field and the magnetic flux. 
flux at the position of the pole head surrounded by the coil reduces to about one half at the bottom of the pole head. The loss of the magnetic flux on the pole head has not been expected in the two-dimensional analysis. The magnetic flux is mostly concentrated on the part of the pole around the exiting coil. The position of the exiting coil on the pole head takes an important meaning for the recording field.

The typical eddy current distributions in the pole head are shown in Fig.4 for $\mathrm{f}=$ $1 \mathrm{MHz}$ and $\sigma=6.25 \times 10^{6} \mathrm{~S} / \mathrm{m}$ and in Fig. 5 for $f^{\circ}=$ $10 \mathrm{MHz}$ and $\sigma=6.25 \times 10^{7} \mathrm{~S} / \mathrm{m}$. The alternating current in the exciting coil has only a real part. The phase of the eddy current leads about 90 degrees against the phase of the exciting current in Fig. 3 and 4 . Therefore, the real part of the eddy current is smaller than its imaginary part. In Fig.4, the imaginary part of the eddy current flows all over the pole head, but in Fig.5, it flows only at the surface of the pole head. It can be explained from the skin depth of the pole metal. The theoretical values of the skin depth in the conditions of Fig. 4 and Fig.5 are 6.37 $\mathrm{mm}$ and $0.64 \mathrm{\mu m}$, respectively. The skin depth in the case of Fig. 5 is very smaller than the thickness of the pole head. The value of the skin depth estimated from Fig.5 is $0.67 \mu \mathrm{m}$, and it agrees closely with the above theoretical value.

The magnetic flux density of the zdirection along the center line of the pole head are shown in Fig.6. The origin of the co-ordinate is on the pole tip surface. In the pole head, the magnetic flux density is remarkably changed by the product of $f$ and $\sigma$. When the skin depth becomes smaller than the thickness of the pole, that is, $f \cdot \sigma>6.25 \times 10^{8} \mathrm{MHz} \cdot \mathrm{S} / \mathrm{m}$, the magnetic flux density around the center of the head vanishes. The magnetic field outside the pole tip, however, is almost independent of the recording frequency and the conductivity of the pole metal. It is found that the eddy current in the single pole head does not affect the magnetic recording f'ield. These results are originated from the open magnetic path peculiar to the single pole type head.

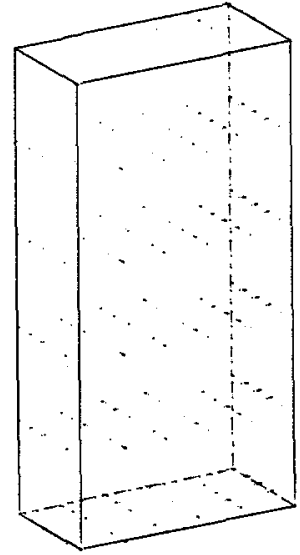

(a) Real part

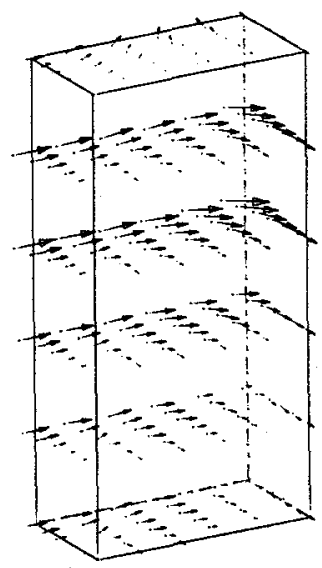

(b) Imaginary part
Fig. 4 Distribution of the eddy current for $f=1 \mathrm{MHz}, \sigma=6.25 \times 10^{6} \mathrm{~S} / \mathrm{m}$. $J_{\max }=1.37 \times 10^{8} \mathrm{~A} / \mathrm{m}^{2}$

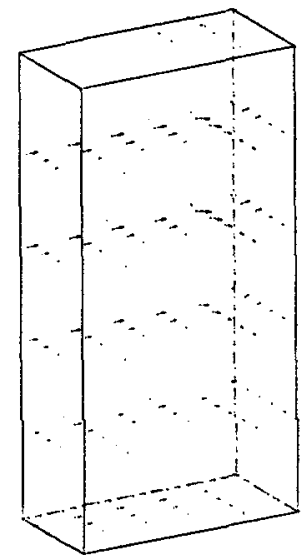

(a) Real part

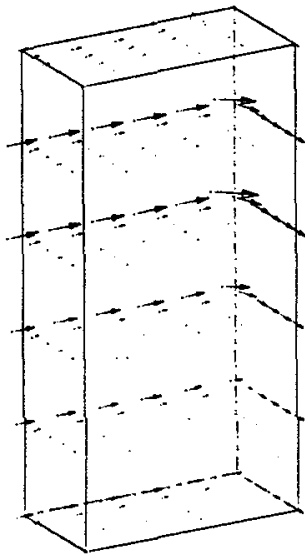

(b) Imaginary part
Fig.5 Distribution of the eddy current for $f=10 \mathrm{MHz}, \sigma=6.25 \times 10^{7} \mathrm{~S} / \mathrm{m}$. $J_{\max }=1.49 \times 10^{10} \mathrm{~A} / \mathrm{m}^{2}$ 


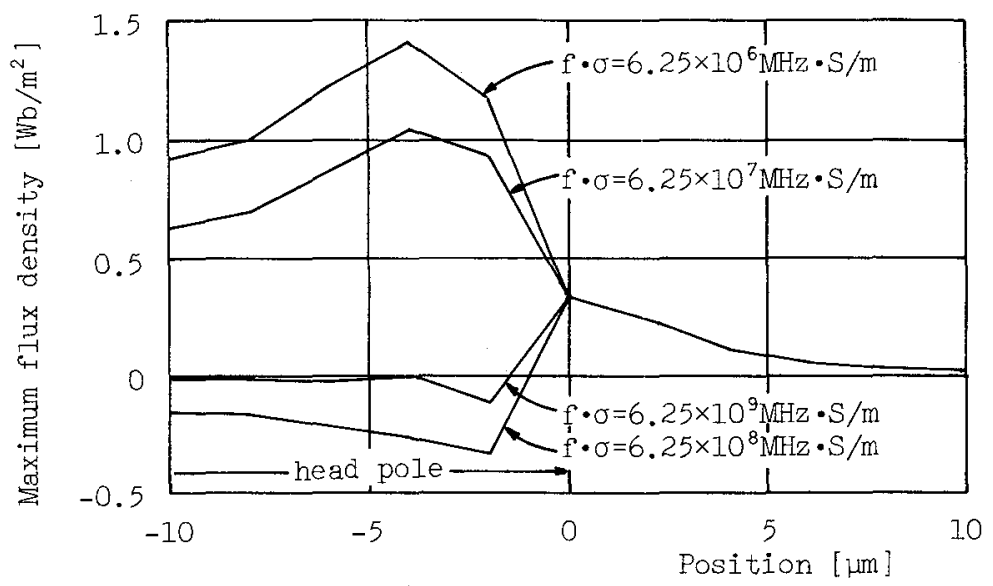

Fig.6 Maximum flux density along the center line of the pole.

\section{CONCLUSION}

The effect of the eddy current of the single pole head on the magnetic recording field has been investigated using a threedimensional finite-element method. The position of the exiting coil on the pole head takes an important meaning for the recording field. Although the large eddy current occurs in the pole head for high frequency recording, the magnetic recording field upon the pole tip surface is not different from the magnetostatic one. These results are originated from the open magnetic path peculiar to the single pole type head.

\section{REFERENCES}

[1] S. Iwasaki and $Y$. Nakamura, "The magnetic field distribution of a perpendicular recording head", IEEE Trans. Magn., Vol.MAG -14, pp. 436-438, Sept. 1978.

[2] J. Hokkyo, I. Saito and S. Satake, "Interaction between head and recording medium in perpendicular recording", IEEE Trans. Magn., Vol.MAG-16, pp.887-889, Sept. 1980. [3] S. Tanabe and S. Iwasaki, rReproducing process of a perpendicular magnetic recording", Trans. IECE of Japan, Vol.J69-C, pp. 312-317, March 1986.
[4] D. A. H. Jacobs, "Generalization of the conjugate gradient method for solving nonsymmetric and complex systems for algebraic equations", CERI Report, RD/1/N 70/80, 1980. 